\title{
Elucidation of the dynamics for hot-spot initiation at nonuniform interfaces of highly shocked materials
}

\author{
Qi An, ${ }^{1}$ Sergey V. Zybin, ${ }^{1}$ William A. Goddard III,,${ }^{1,}$ Andres Jaramillo-Botero, ${ }^{1}$ Mario Blanco, ${ }^{1}$ and Sheng-Nian Luo ${ }^{2}$ \\ ${ }^{1}$ Materials and Process Simulation Center, California Institute of Technology, Pasadena, California 91125, USA \\ ${ }^{2}$ Los Alamos National Laboratory, Los Alamos, New Mexico 87545, USA
}

(Received 9 August 2011; revised manuscript received 3 October 2011; published 7 December 2011)

\begin{abstract}
The fundamental processes in shock-induced instabilities of materials remain obscure, particularly for detonation of energetic materials. We simulated these processes at the atomic scale on a realistic model of a polymer-bonded explosive $(3,695,375$ atoms/cell) and observed that a hot spot forms at the nonuniform interface, arising from shear relaxation that results in shear along the interface that leads to a large temperature increase that persists long after the shock front has passed the interface. For energetic materials this temperature increase is coupled to chemical reactions that lead to detonation. We show that decreasing the density of the binder eliminates the hot spot.
\end{abstract}

DOI: 10.1103/PhysRevB.84.220101

PACS number(s): $62.50 . \mathrm{Ef}, 82.40 . \mathrm{Fp}, 47.40 . \mathrm{Nm}$

The interaction of shock waves with nonuniform interfaces plays an essential role in the interfacial instabilities in inertial confinement fusion (ICF), in shock-induced RichtmyerMeshkov instabilities (RMIs), and in detonation in heterogeneous polymer-bonded explosives (PBXs). For detonation, it is generally accepted that hot spots form during the development of instabilities as shock waves pass through the interface or other defects. ${ }^{1-4}$ Despite numerous experimental and theoretical studies, the fundamental processes involved remain controversial. This is due to the complex environment and coupling of thermal, chemical, and mechanical degree of freedom, which is extremely difficult to unravel experimentally. It has also been very difficult theoretically to include the reactive processes involved and yet cover the enormous size and time scales intrinsic to the phenomena.

To discover the origin of shock-induced hot-spot formation, we carried out reactive dynamics (RD) using the ReaxFF reactive force field on a realistic model of a real polymerbonded explosive PBX N-106. Energetic materials (EMs) are essential for applications ranging from rocket engines, to building and dam construction, and to armaments. Generally the EM is bound together in a matrix of polymer elastomers to form the PBX that can be molded into various shapes, while providing some control in resisting unintentional detonation due to shocks or friction. Unfortunately, current generations of PBXs are sensitive to accidental detonation, despite many attempts to control the safety by improvements in materials and manufacturing practices. Here we use the ReaxFF reactive force field to examine the effect of shocks on realistic models of polymer-bonded explosives, where we use these simulations to extract the mechanism of hot-spot formation. Then, based on this model, we predict how to change the system to reduce the hot spot, and carry out simulations to validate this prediction.

ReaxFF has now been established to provide nearly the accuracy of quantum calculation in the various reaction barriers and rates while providing a computational efficiency nearly that of ordinary molecular dynamics (MD) with ordinary force fields (FFs), enabling us to study the complex processes involved in interfacial instabilities at the atomic scale, providing insights on such phenomenon. Thus ReaxFF provides the possibility of realistic simulations to probe the atomistic mechanism controlling detonation, ${ }^{5-12}$ and leads to an accurate description of the complex chemistry of cyclotrimethylene trinitramine (RDX) under shock-loading conditions, ${ }^{6}$ and similar calculations on polyethylene (PE) and poly(4-methyl-1-pentene) polymer lead to good agreement with the experimental Hugoniot. ${ }^{9}$

It is generally accepted that detonation of PBXs is initiated at hot spots, but the mechanism responsible for hot-spot ignition is not clear. ${ }^{13}$ Previous simulations ${ }^{14-17}$ in relatively small molecular crystals and atomic crystals containing voids postulated that void collapse and nanojets in voids are the source of hot-spot formation.

In order to develop an atomic-scale understanding of the nature of hot-spot formation of PBX materials we used ReaxFF RD simulations to examine the effect of shocks on the materials configuration in Fig. 1, which involves a sawtooth interface between the RDX and the polymer [hydroxyl-terminated polybutadiene (HTPB)]. This system is $54 \mathrm{~nm}$ thick in the shock direction $(x)$ with a period of $27 \mathrm{~nm}$ along the sawtooth direction $(y)$, and is uniform with a periodic length of $25 \mathrm{~nm}$ in the $z$ direction, leading to a simulation cell with 3695375 independent atoms. For shock velocities of 2.5 and $3.5 \mathrm{~km} / \mathrm{s}$ it takes $\sim 10$ ps for the shock wave to traverse the interface. Such a simulation cell and time interval is far beyond the capabilities of current quantum mechanics (QM) methods but can be treated using the QM-based ReaxFF reactive force field.

Our simulations model the PBXN-106 material, which consists of cyclotrimethylene trinitramine (RDX) crystals bound together using HTPB- and isophorone diisocyanate (IPDI)-based polyurethane rubber. HTPB (see Fig. S1 of the Supplemental Material ${ }^{18}$ ) is a copolymer, containing $80 \%$ trans- and cis-butadiene monomers with $20 \%$ 1,2-vinyl monomers, with the chains terminated by hydroxyl groups. Our MD simulations use $100 \%$ trans monomers, leading to a molecular weight of 1518 Da per chain. A plasticizer agent di-octyl adipate (DOA) is added ( $40 \%$ by weight) to the binder to improve casting properties. To determine the conformational state of the polymer at $300 \mathrm{~K}$ and $1 \mathrm{~atm}$ pressure, we used the cohesive energy density (CED) Monte Carlo method ${ }^{19,20}$ designed to predict accurate cohesive energy densities of 


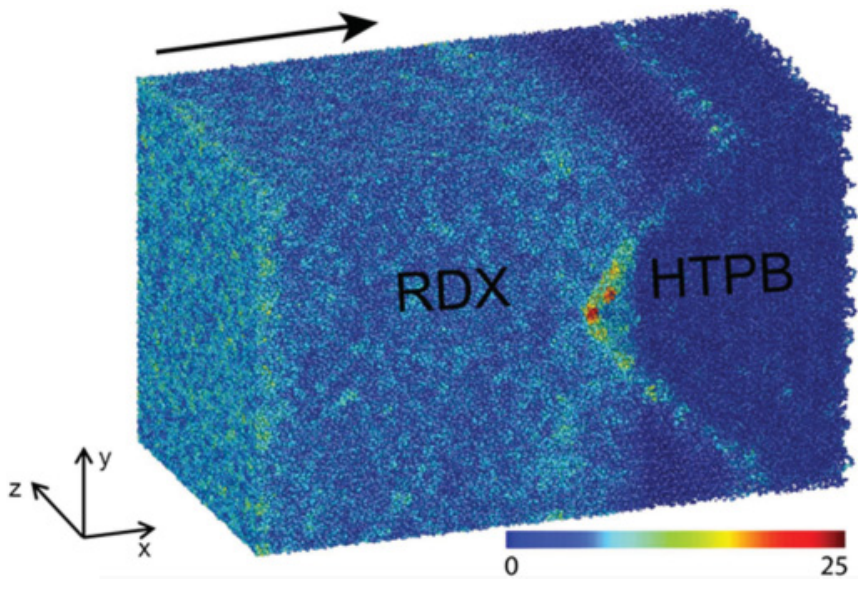

FIG. 1. (Color online) Snapshot of PBX during shock loading at $U_{p}=2.5 \mathrm{~km} / \mathrm{s}$ (for $6.0 \mathrm{ps}$ ). The shading is based on the total slip in angstroms. This system is $54 \mathrm{~nm}$ thick in the shock direction $(x)$, with a period of $27 \mathrm{~nm}$ along the sawtooth direction $(y)$, and is uniform with a periodic length of $25 \mathrm{~nm}$ in the $z$ direction. The arrow indicates shock direction.

polymers (CED combines repeated cycles of temperature annealing and quenching simultaneous with density annealing and quenching to ensure that polymer chains are fully equilibrated). We used CED to build ten independent samples of each polymer within a unit cell containing two independent molecular chains and eight DOA molecules. We chose the mass ratio of binder over DOA to be 1.03 , which leads to the optimum viscosity and compression strength. ${ }^{21}$ Each chain contains ten HTPB repeat units connected via one IPDI crosslinking molecule to four terminal HTPB repeat units per end. The initial conformation was based on rotational isomeric states (RISs) statistics to determine the dihedral angle values for each chain and its repeating units. The torsional barriers for the RIS and the charges (Mulliken) were determined [from Becke three-parameter Lee-Yang-Parr (B3LYP) hybrid density functional calculations using the $6-311 \mathrm{G}^{* *}$ basis set] on a polybutadiene trimer. The same level of $\mathrm{QM}$ calculations was also performed on the IPDI and DOA to determine the charges and structures for the binder model. These QM results and model preparation procedure are provided as part of the Supplemental Material. ${ }^{18}$

To simulate the propagation of shock waves in the PBX, we impacted the two-dimensionally (2D) periodic PBX slab onto a Lennard Jones 9-3 wall. The shock direction (x) was set to be finite. The slab was thermally equilibrated at $\mathrm{T}=300 \mathrm{~K}$ for $4 \mathrm{ps}$ and then propelled at the desired impact velocity by adding a corresponding translational velocity on top of the thermal component. This procedure produces a shock wave with an average particle velocity $(U p)$ equal to the impact velocity, propagating into the slab from the RDX side. Here we explore the shock response of the nonuniform interface in the PBX at $U p=2.5$ and $3.5 \mathrm{~km} / \mathrm{s}$ via adiabatic MD to follow the dynamic evolution of the system, using a time step of $0.1 \mathrm{fs}$ to integrate the equations of motion, for up to $10 \mathrm{ps}$.

Considering the heterogeneities normal to the shock direction $(x)$, we partition the simulation cell into fine bins along the $x, y$ directions and average all physical properties of interest within each bin, including stress $\left(\sigma_{i j}\right)$ and temperature $(T)$ profiles in the $x y$ plane at different stages of compression $(i, j=1,2$, and 3 , corresponding to $x, y$, and $z$, respectively.) The atomic stress is obtained from the atomic virial. ${ }^{22}$ The stress calculation is based on the center of mass (COM) of each molecule to eliminate binning effects. The bin width is $\sim 1 \mathrm{~nm}$ $\times 1 \mathrm{~nm}$ in the xy plane. The von Mises shear stress $\tau$ is also computed as the maximum shear stress, as $2 \tau=\sigma_{x x}-\left(\sigma_{y y}+\right.$ $\left.\sigma_{z z}\right) / 2$, under the assumption of $\sigma_{y y}=\sigma_{z z}$, which is true in our simulations.

To track the chemical processes as the shock wave propagates through the PBXs, we analyze the fragments resulting from the corresponding ReaxFF MD trajectories based on a time-windowed average of bond orders. Combining the fragment analysis results and the two-dimensional binning

I Temperature (K) for Up $=2.5 \mathrm{~km} / \mathrm{s}$ (a) $5.0 \mathrm{ps}$

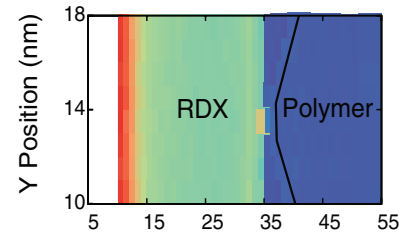

(c) $6.0 \mathrm{ps}$

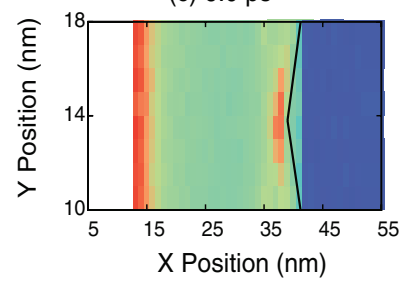

II Shear Stress (GPa) for Up $=2.5 \mathrm{~km} / \mathrm{s}$

(e) $4.5 \mathrm{ps}$

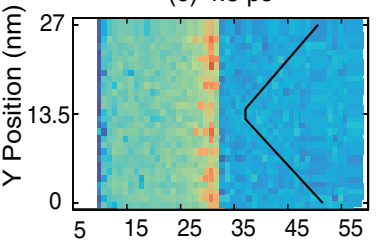

(g) $5.5 \mathrm{ps}$

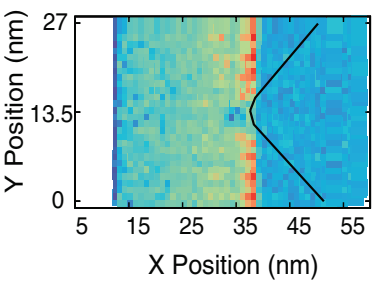

(b) $5.5 \mathrm{ps}$

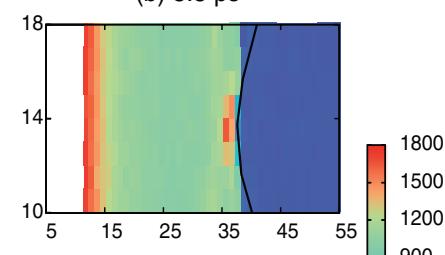

(d) $8.0 \mathrm{ps}$

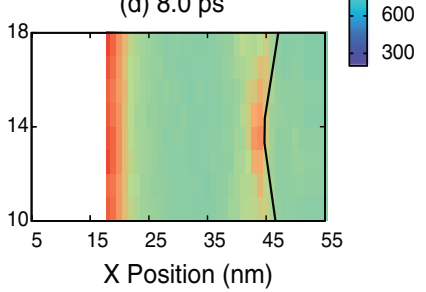

800
500
200
00
00
00
FIG. 2. (Color online) Time evolution of temperature and von Mises shear stress for $U_{p}=2.5 \mathrm{~km} / \mathrm{s}$ at various stages during the shock compression. The solid line represents the interface position between the RDX and the binder. Shock wave propagates from left (RDX) to right (binder). (e) $4.5 \mathrm{ps,} \mathrm{shock} \mathrm{wave} \mathrm{has} \mathrm{not} \mathrm{propagated}$ to polymer; (a),(f) $5.0 \mathrm{ps,} \mathrm{shock} \mathrm{wave} \mathrm{just} \mathrm{reaches} \mathrm{the} \mathrm{interface;}$ (b),(g) 5.5 ps, adiabatic shear localization in a small triangle interface region; (c),(h) 6.0 ps, shock wave has passed through the hot-spot region; (d) 8.0 ps, shock wave has passed through the interface. 
analyses, we examined the chemistry in the xy plane at different stages of shock loading. To examine the atomic local shear, we use maximum relative displacement (MRD), ${ }^{23,24}$ which is defined as $\mathbf{s}_{i}=\mathbf{x}_{i j}-\mathbf{X}_{i j}:\left|\mathbf{x}_{i j}-\mathbf{X}_{i j}\right|_{\text {max }}$. Here the $\mathrm{X}_{i j}$ and $\mathrm{X}_{i j}$ vectors correspond to the difference between the current and reference configurations for atoms $i$ and $j$ (slipped neighbors of atom $i$ ), respectively. The reference configurations are taken to be the preshock structures.

Figures 2(a)-2(d) show the 2D profile of temperature $T$ and shear stress $\tau$ at various stages of compression for $U_{p}=$ $2.5 \mathrm{~km} / \mathrm{s}$. The temperature at the angular tip interface region increases by $\sim 400 \mathrm{~K}$ compared to other shocked regions, once the shock wave has passed through, leading to formation of a hot spot. The hot-spot region expands into the explosive along the interface as the shock wave continues to propagate. The shock wave converges as it propagates in the binder due to the wave refraction, as it passes through the nonuniform interface. This shock-wave convergence leads to a small, second highest temperature region $\sim 50 \mathrm{~K}$ higher (see Fig. S5 in the Supplemental Material ${ }^{18}$ ) to the right of the hot-spot region in the binder. This hot spot also appeared at higher shock velocities. ${ }^{18}$

To understand the mechanism underlying the hot-spot formation found in our study, we traced the von Mises shear stress at different stages. Upon shock arrival to the interface, we find reduction of shear stresses at the angular tip regions as manifested in Fig. 2(g). This arises from rapid relaxation of the stress at the tip by the more compressible polymer, possibly combined with the convergence of the shock wave at the tip, interfacial friction between RDX and binder, and perhaps the collapse of tiny nanovoids at the angular tip. This shear localization at the angular tip leads to nucleation of the hot spot. The stress decreases rapidly in the hot-spot region, leading to large local shear deformations as shown in Fig. 2(h). This small hot-spot region expands rapidly as the shock wave propagates, causing additional shear localization near the interface in the explosive and an additional increase in the local temperature. Since the time scale is only picoseconds, we consider this to be an adiabatic shear localization process. The expansion in the shear localized region is depicted in Figs. 2(g) and 2(h). The MRD analysis in Fig. 1 shows that the regions with maximum local deformation correspond to the maximum local shear stress relaxation, and to the hot spot. Since the shock wave propagates from high-density (RDX) explosives to a low-density (binder) polymeric matrix, the reflective waves from the interface will decrease the stress in the explosive, which tends to suppress the hot-spot formation as discussed below. Thus we find that the hot spot forms in our system as a compromise between shear localization and reflective wave relaxation in the angular tip and interface regions. Our atomically based hot-spot ignition mechanism provides an understanding that differs from previously proposed mechanisms ${ }^{25}$ involving adiabatic compression of a trapped gas, cavity collapse including viscous or plastic heating of the surrounding matrix material, friction between the impacting surfaces, among other phenomena.

In order to follow the chemical processes as the shock wave propagates through the interface of the RDX and the binder, we plot the distribution of $\mathrm{NO}_{2}$ production in the $x y$ plane since $\mathrm{NO}_{2}$ dissociation is the main chemical product for $U p$ below 4 km/s (see Ref. 6). Figure 3 shows the time evolution of $\mathrm{NO}_{2}$ for $U_{p}=2.5$ and $3.5 \mathrm{~km} / \mathrm{s}$. We find few reactions for $U_{p}=2.5 \mathrm{~km} / \mathrm{s}$, except those occurring in the hot-spot region. The plastic shear deformation in the hot-spot region (a) $U p=2.5 \mathrm{~km} / \mathrm{s}$, time $=6 \mathrm{ps}$

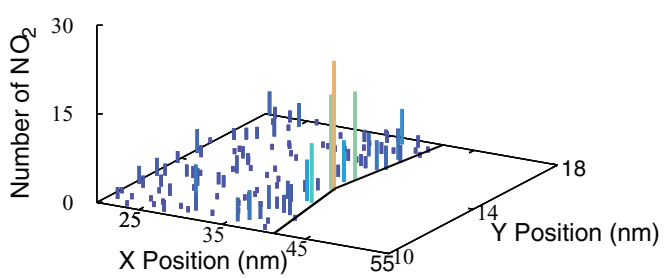

(c) $U p=3.5 \mathrm{~km} / \mathrm{s}$, time $=4.5 \mathrm{ps}$

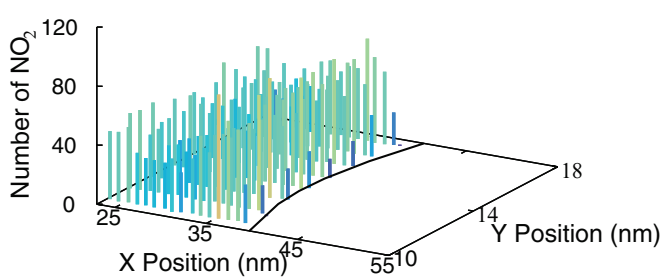

(b) $U p=2.5 \mathrm{~km} / \mathrm{s}$, time $=8 \mathrm{ps}$

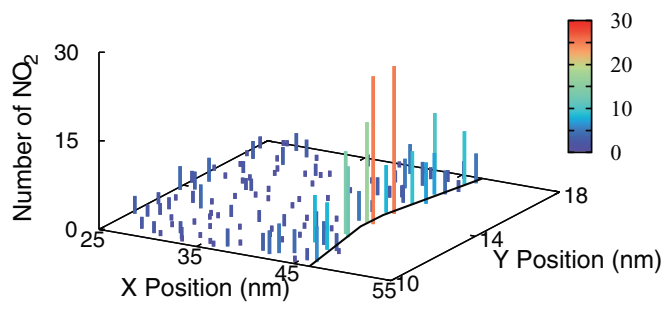

(d) $U p=3.5 \mathrm{~km} / \mathrm{s}$, time $=6.5 \mathrm{ps}$

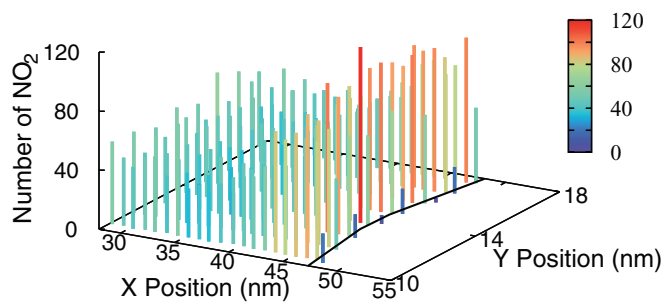

FIG. 3. (Color online) 2D distribution and time evolution of chemistry $\left(\mathrm{NO}_{2}\right.$ formation) for various $U_{p}$. (a) $U_{p}=2.5 \mathrm{~km} / \mathrm{s}$ at $t=6.0 \mathrm{ps}$ : The chemical reaction has occurred mainly in the hot-spot region. (b) $U_{p}=2.5 \mathrm{~km} / \mathrm{s}$ at $t=8.0 \mathrm{ps}$ : Additional chemical reactions have occurred and a small amount of $\mathrm{NO}_{2}$ has diffused to the polymer region (the right region of the solid line in the $x y$ plane). (c) $U_{p}=3.5 \mathrm{~km} / \mathrm{s}$ at $t=4.5$ ps: The chemical reaction occurs in the shocked regions but much more occur in the hot-spot region. (d) $U_{p}=3.5 \mathrm{~km} / \mathrm{s}$ at $t=6.5 \mathrm{ps}$ : We see diffusion of $\mathrm{NO}_{2}$ into the polymer region. 


\section{Temperature $(\mathrm{K})$ for $U p=3.5 \mathrm{~km} / \mathrm{s}$}
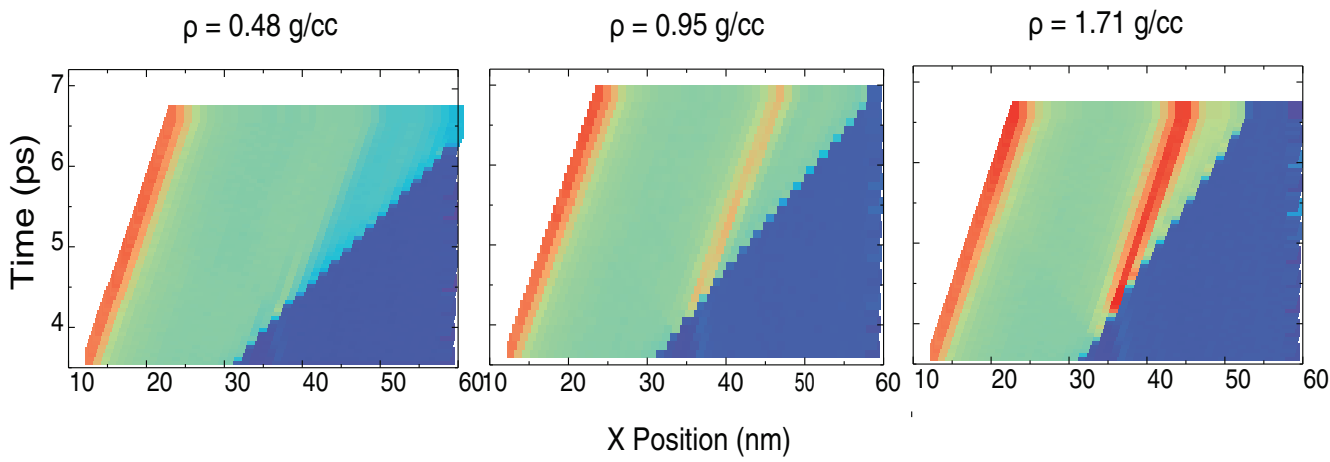

0500100015002000250030003500

Shear Stress (GPa) for Up $=3.5 \mathrm{~km} / \mathrm{s}$

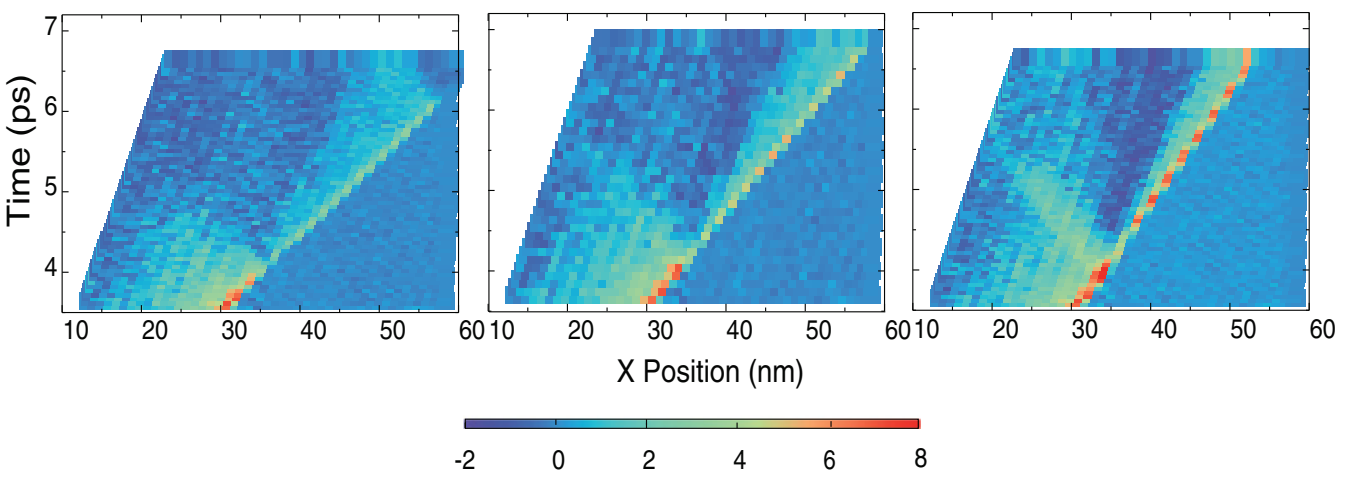

FIG. 4. (Color online) The dependence of hot-spot formation (temperature and shear stress) on the density of the polymer binder at a shock velocity of $3.5 \mathrm{~km} / \mathrm{s}$. The middle $\left(0.95 \mathrm{~g} / \mathrm{cm}^{3}\right)$ is normal density. Thus using of a polymer with half the normal density prevents hot-spot formation. This is due to the improved interface impedance which allows reflective rarefaction wave relaxation. In contrast, increasing the binder density enhances the hot spot due to the reflective shock waves reflected back into the RDX.

produces enough energy for N-N bond excitations, leading to increased $\mathrm{NO}_{2}$ formation. The same phenomenon occurs for $U_{p}=3.5 \mathrm{~km} / \mathrm{s}$. The chemical reactions occurring in the hotspot region release additional energy, expanding it to induce even more shear deformation. Under the coupling effects of local shear relaxation, increased chemical reactions, increased local temperature, and the reflective wave relaxation, the hot spot expands until detonation (or at lower input shock velocity it can disappear).

Our hot-spot ignition mechanism suggests that use of a binder with lower density would lead to decreased hot-spot formation and hence a less sensitive EM for propulsion and explosives applications. To test this idea we changed the binder density to half the original value $\left(0.95 \mathrm{~g} / \mathrm{cm}^{3}\right)$, to approximately twice the value by scaling the atomic mass. We carried out shock simulations at $U_{p}=3.5 \mathrm{~km} / \mathrm{s}$. To analyze the hot-spot behavior we display a 2-nm segment in the $y$ direction for a one-dimensional binning analysis during shock compression. Figure 4 shows the temperature-time and shear stress-time diagrams from $3.6 \mathrm{ps}$, at which the shock wave has not reached the interface region. The temperature-time diagram shows that the hot spot is dramatically increased for the high-density binder, but it disappears for the halfdensity case $\left(0.48 \mathrm{~g} / \mathrm{cm}^{3}\right)$. Indeed, this low-density binder suppressed hot-spot formation due to the relaxation of the hot spot.

Previous studies ${ }^{26,27}$ showed that a second shock wave (double shock) or a rarefaction wave will be reflected back into the original medium as the shock wave travels through interface. These refraction wave patterns are determined by the shock impedance, which is a function of the incident wave angle, the sound speed at the shocked materials interfaces, and the stresses in the two regions. As shown in the normal stress-time behavior $\left(\sigma_{11}-t\right.$, see Fig. S6 of the Supplemental Material ${ }^{18}$ ), a high-density polymer matrix leads to a reflected shock wave due to the higher shock impedance at the interface, while the low-density polymer matrix leads to a rarefaction wave. The von Mises shear stress diagram in Fig. 4 shows the same character: A high shear stressed reflective wave forms for the high-density case, a modest one for normal density, and complete disappearance for the low-density case (here the reflective wave relaxes the system, avoiding the hot spot). These results indicate that the hot spot arises from the complex variation of shock impedance at this nonuniform interface. We also note from the $x$ - $t$ diagram that the shock wave propagates at a slower speed through the high-density binder matrix.

These results suggest that a critical design strategy for developing insensitive EM for propulsion and explosives is 
to use a binder with significantly lower mechanical impedance than that of EM. Thus, merely reducing the density of the polymer by a factor of 2 eliminates the hot spot.

Summarizing, we used reactive dynamics (RD) to examine the mechanical, chemical, and thermal response of mechanically shocked polymer-boned explosives (PBXs) by using a realistic model of a nonuniform interface with 3695375 independent atoms. We observe that a hot spot develops at the first point at which the shock wave encounters the lower-density polymer at the nonuniform interface between the explosive and elastomer. Our analysis shows that the hot spot arises from adiabatic shear localization, which leads to a large temperature increase that persists long after the shock front has passed the interface, followed for energetic materials, by coupling to chemical reactions ( $\mathrm{NO}_{2}$ and $\mathrm{HONO}$ dissociation) that ultimately leads to detonation.

We should emphasize the sequence of events here: (a) Shock at the asperity of a nonuniform interface of heterogeneous materials leads to shear relaxation that causes shear stresses along the nonuniform interface. (b) These shear stress relaxations result in energy deposition at the interface that leads to a local significant increase in temperature. (c) This increase in temperature leads to bond breaking in energetic materials, which leads eventually to detonation (which may be premature).

For nonenergetic materials we also expect the first two phenomena of local shear stress and temperature increase. Thus for nonenergetic materials we can expect that the hot spot may cause local melting and defect generation that would accelerate failure and perhaps instabilities (e.g., RichtmyerMeshkov).

These results suggest that a critical design strategy in developing insensitive EM for propulsion and explosives is to use a binder with a much lower density (1/3 that of the explosive). We tested this by modifying the polymer system to have half the normal density (1/3 that of the explosive) and showed that no hot spot develops using the same shock conditions.

All computations were carried out on the Army HPC systems; we thank Betsy Rice and Larry Davis for assistance. Personnel were supported by ARO (W911NF-05-1-0345 and W911NF-08-1-0124) and by ONR (N00014-09-1-0634). *wag@wag.caltech.edu

${ }^{1}$ J. P. Dear, J. E. Field, and A. J. Walton, Nature (London) 332, 505 (1988).

${ }^{2} \mathrm{R}$. Chéret, Detonation of Condensed Explosives (Springer, New York, 1993).

${ }^{3}$ F. P. Bowden and A. D. Yoffe, Initiation and Growth of Explosions in Liquids and Solids (Cambridge University Press, Cambridge, UK, 1952).

${ }^{4}$ P. Maffre and M. Peyrard, Phys. Rev. B 45, 9551 (1992).

${ }^{5}$ K. I. Nomura, R. K. Kalia, A. Nakano, P. Vashishta, A. C. T. van Duin, and W. A. Goddard III, Phys. Rev. Lett. 99, 148303 (2007).

${ }^{6}$ A. Strachan, A. C. T. van Duin, D. Chakraborty, S. Dasgupta, and W. A. Goddard III, Phys. Rev. Lett. 91, 098301 (2003).

${ }^{7}$ A. C. T. van Duin, S. Dasgupta, F. Lorant, and W. A. Goddard III, J. Phys. Chem. A 105, 9396 (2001).

${ }^{8}$ A. Strachan, E. M. Kober, A. C. T. van Duin, J. Oxgaard, and W. A. Goddard III, J. Chem. Phys. 122, 054502 (2005).

${ }^{9}$ T. R. Mattsson, J. M. D. Lane, K. R. Cochrane, M. P. Desjarlais, A. P. Thompson, F. Pierce, and G. S. Grest, Phys. Rev. B 81, 054103 (2010).

${ }^{10}$ L. Z. Zhang, S. V. Zybin, A. C. T. van Duin, S. Dasgupta, W. A. Goddard III, and E. M. Kober, J. Phys. Chem. A 113, 10619 (2009).

${ }^{11}$ N. Rom, S. V. Zybin, A. C. T. van Duin, W. A. Goddard III, Y. Zeiri, G. Katz, and R. Kosloff, J. Phys. Chem. A 115, 10181 (2011).

${ }^{12}$ S. V. Zybin, W. A. Goddard III, P. Xu, A. C. T. van Duin, and A. P. Thompson, Appl. Phys. Lett. 96, 081918 (2010).
${ }^{13}$ J. E. Field, Acc. Chem. Res. 25, 489 (1992).

${ }^{14}$ B. L. Holian, T. C. Germann, J. B. Maillet, and C. T. White, Phys. Rev. Lett. 89, 285501 (2002).

${ }^{15}$ J. W. Mintmire, D. H. Robertson, and C. T. White, Phys. Rev. B 49, 14859 (1994).

${ }^{16}$ K. Nomura, R. K. Kalia, A. Nakano, and P. Vashishta, Appl. Phys. Lett. 91, 183109 (2007).

${ }^{17}$ T. Hatano, Phys. Rev. Lett. 92, 015503 (2004).

${ }^{18}$ See Supplemental Material at http://link.aps.org/supplemental/ 10.1103/PhysRevB.84.220101 for Construction of the PBX model and additional details on the shock simulations.

${ }^{19}$ I. Cozmuta, M. Blanco, and W. A. Goddard III, J. Phys. Chem. B. 111, 3151 (2007).

${ }^{20}$ M. Belmares, M. Blanco et al., J. Comput. Chem. 25,1814 (2004).

${ }^{21}$ J. S. Gharia, R. K. Sinha, V. V. Tadas, Vinay Prakash, and V. K. Phadke, Defence Sci. J. 48, 125 (1998).

${ }^{22}$ J. F. Lutsko, J. Appl. Phys. 65, 2991 (1989).

${ }^{23}$ J. A. Zimmerman, C. L. Kelchner, P. A. Klein, J. C. Hamilton, and S. M. Foiles, Phys. Rev. Lett. 87, 165507 (2001).

${ }^{24} \mathrm{C}$. Brandl, Ph.D. thesis, École Polytechnique Fédérale de Lausanne, 2009.

${ }^{25}$ J. E. Field, N. K. Bourne, S. J. P. Palmer, S. M. Walley, J. Sharma, and B. C. Beard, Philos. Trans. R. Soc. London A 339, 269 (1992).

${ }^{26}$ H. Polachek and R. J. Seeger, Phys. Rev. 84, 922 (1951).

${ }^{27}$ L. F. Henderson, J. Fluid Mech. 40, 719 (1970). 